Mappemonde

Revue trimestrielle sur l'image géographique et les

formes du territoire

$131 \mid 2021$

Varia

\title{
Explorer la 2D/3D interactive pour représenter des flux
}

Quentin Nahélou

CpenEdition

Journals

Édition électronique

URL : https://journals.openedition.org/mappemonde/6308

DOI : $10.4000 /$ mappemonde.6308

ISSN : 1769-7298

Éditeur

UMR ESPACE

Référence électronique

Quentin Nahélou, «Explorer la 2D/3D interactive pour représenter des flux », Mappemonde [En ligne] 131 | 2021, mis en ligne le 08 juillet 2021, consulté le 14 juillet 2021. URL : http://

journals.openedition.org/mappemonde/6308; DOI : https://doi.org/10.4000/mappemonde.6308

Ce document a été généré automatiquement le 14 juillet 2021.

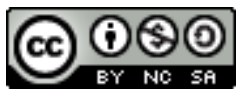

La revue Mappemonde est mise à disposition selon les termes de la Licence Creative Commons Attribution - Pas d'Utilisation Commerciale - Partage dans les Mêmes Conditions 4.0 International. 


\title{
Explorer la 2D/3D interactive pour représenter des flux
}

\author{
Quentin Nahélou
}

\section{Contexte}

1 Les données de flux sont des données complexes, leur représentation cartographique n'est jamais une chose aisée et l'innovation dans cet exercice est délicate, tant leur représentation peut être régie par des normes de sémiologie strictes. Néanmoins, les possibilités de cartographies dynamiques permettent aujourd'hui d'explorer de nouveaux modes de représentation de cette donnée complexe, de la 2D interactive jusqu'à la 3D, en passant par l'animation.

2 Ainsi cette application, et plus particulièrement la première carte interactive, a été voulue comme un travail exploratoire autour de l'utilisation des 2D/3D interactives pour représenter des flux.

\section{Description}

3 Cette application cible une ville aéroportuaire choisie par l'utilisateur pour y étudier les dynamiques en termes de transport de passagers. Elle se compose de plusieurs visualisations :

4 Une première carte interactive (figure 1) qui représente l'exploration spatiotemporelle du trafic aérien de la ville choisie. Sur le côté droit, un graphique de type histogramme renseigne sur les stocks des différents types de flux pour l'année indiquée. En scrollant sur la page, un court texte décrivant les dynamiques de ces flux depuis 1999 apparaît. 
Figure 1. Exploration visuelle du trafic aérien

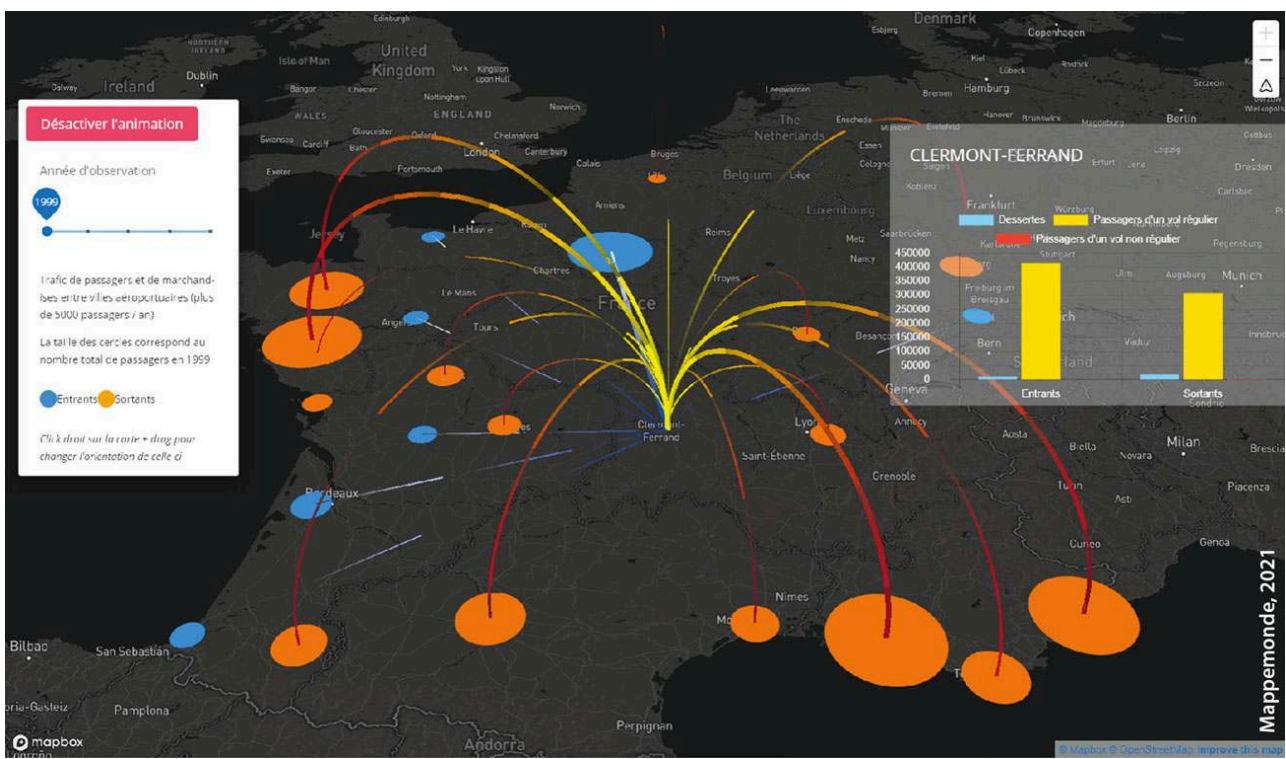

5 La seconde carte (figure 2) renseigne quant à elle sur des stocks de passagers, ainsi que sur des mouvements d'avions, de 2010 à 2020, par mois. Un graphique de type linéaire sur le côté droit, vient informer de l'évolution dans l'année des stocks sélectionnés, mais aussi de l'évolution de ces derniers durant l'année n-1.

Figure 2. Exploration de la fréquentation

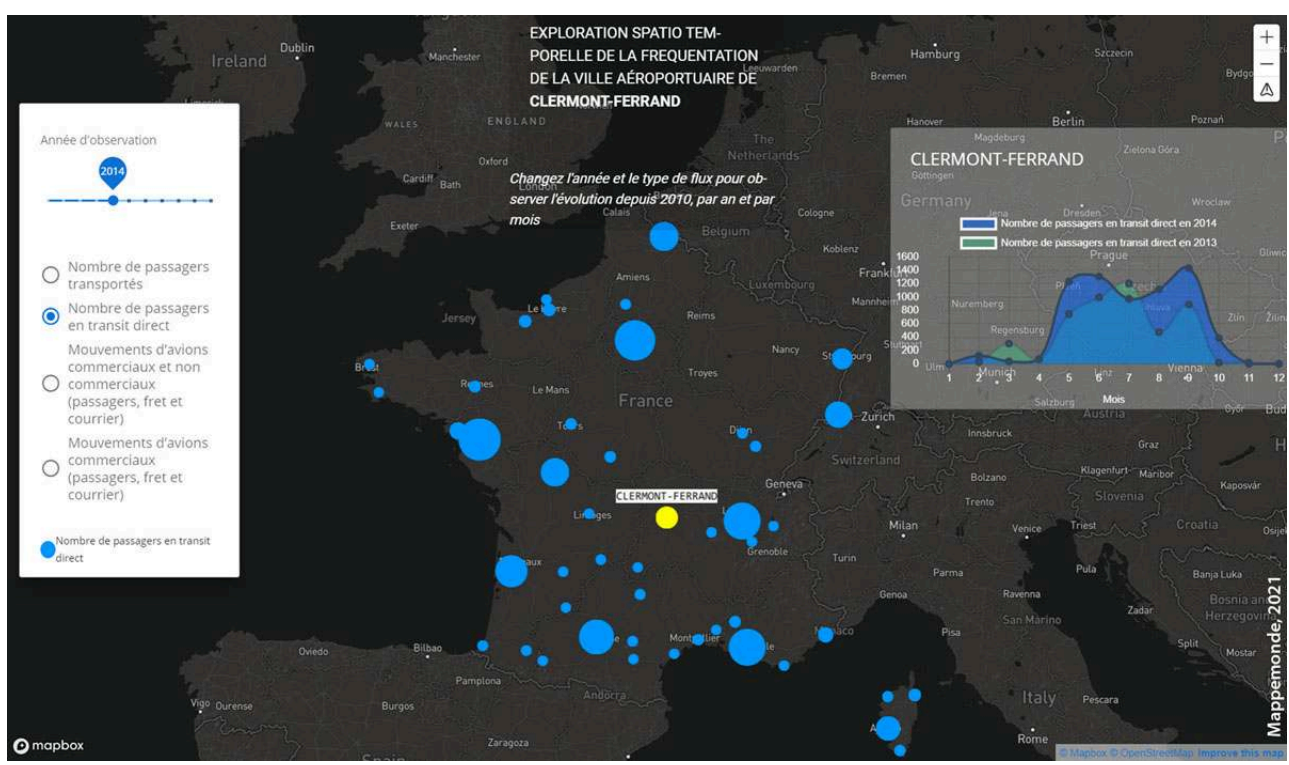

6 Enfin, la dernière partie de cette application (figure 3) est un graphique de type Bubble Plot de synthèse. Il identifie la position de la ville choisie, dans les dynamiques de transport de passagers des villes aéroportuaires de France Métropolitaine, en croisant l'évolution du nombre de passagers transportés en 2019 et en 2020. 
Figure 3. Dynamique des villes aéroportuaires (bubble plot)

Synthèse des dynamiques des villes aéroportuaires de France (hors Paris)

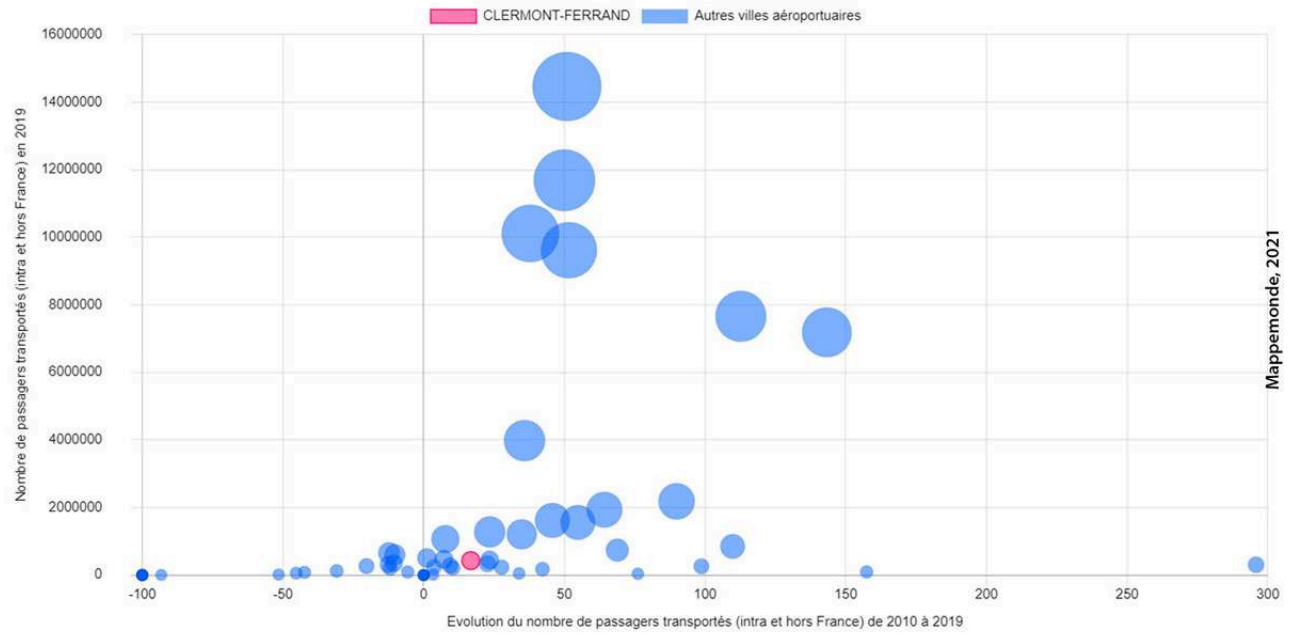

Avec une évolution du nombre de passagers transportés de $+16.74 \%$ entre 2010 et 2019 pour atteindre un nombre de passagers transportés de 426779 en 2019. On peut dire que la dynamique de la ville aéroportuaire de CLERMONT-FERRAND en terme de trafic de passagers est moyenne

\section{Technologies}

$7 \quad$ L'application est développée avec le framework JavaScript Vue.js. Elle est statique et ne possède donc pas de partie BackEnd. Les cartes interactives ont été créées à partir du framework Deck.gl avec la bibliothèque Mapbox.gl. Les graphiques ont, quant à eux, été développés avec la bibliothèque Chart.js.

8 La préparation des données a entièrement été effectuée en SQL avec PostgreSQL. Un petit script $\mathrm{R}$ a été utilisé pour générer les statistiques nécessaires au calcul des textes présents sur l'application.

\section{Apports à la géo-datavisualisation}

Pour assurer la lisibilité de la représentation des données de flux, cette application propose plusieurs types de rendus (cartes, graphiques et textes) pour une exploration complémentaire des informations. L'application présente plusieurs manières de sélectionner l'information à représenter, pour gagner en lisibilité et enrichir l'expérience et l'analyse des cartes.

L'idée générale est de pouvoir raconter la même histoire sur les différentes villes aéroportuaires, de pouvoir les comparer. C'est pourquoi l'utilisateur est amené à choisir une ville au début de son expérience (figure 4). 


\section{QUELLES DYNAMIQUES POUR LES VILLES AÉROPORTUAIRES DE FRANCE?}

Zoom sur la ville de CLERMONT-FERRAND

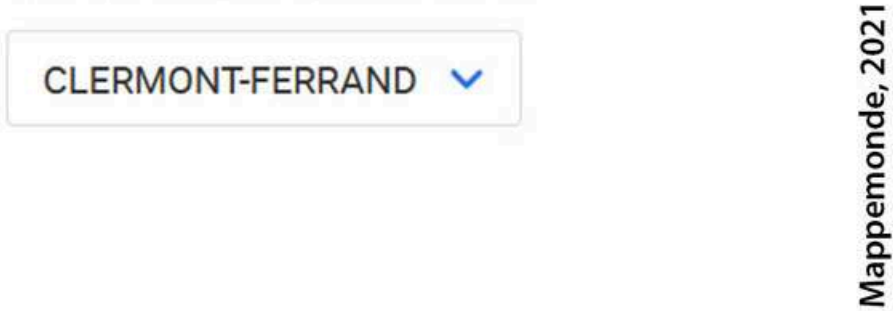

\section{SCROLL TO CONTINUE}

11 Chaque ville dispose cependant du commentaire associé à ses informations, qui sont également temporelles.

12 Le choix d'utiliser les nouvelles technologies de visualisation des flux en 2D/3D est une prise de risque importante, surtout pour réaliser une application en temps limité. Les problèmes techniques qui demeurent auraient pu être résolus avec plus de temps.

\section{Limites}

La plus grande des limites de l'application réside dans la mauvaise compréhension d'un jeu de données. En effet, les flux n'étaient pas orientés, de fait leur modélisation sur la première cartographie est fausse. Il aurait fallu n'utiliser qu'un seul figuré non orienté.

Une autre limite de cette application est dans l'accessibilité des technologies utilisées. En effet, si la préparation de la donnée en SQL est une compétence acquise par bon nombre de géomaticiens, cartographes et experts de la géo-datavisualisation, le développement web reste une compétence qui peut parfois être plus secondaire, or elle est la base même de cette application. Et si des bibliothèques de cartographie web comme Leaflet ou OpenLayers sont des institutions nourries d'exemples fournis par une communauté importante, d'autres, comme DeckGL qui est utilisée ici, le sont beaucoup moins, ainsi leur apprentissage et la résolution de problèmes deviennent bien plus complexes, d'autant plus dans un temps limité. Enfin, l'application est très demandeuse de ressources, notamment du côté du CPU. En effet, la solution d'animation qui a été utilisée ici n'a pas eu le temps d'être optimisée, ainsi un bouton pour désactiver l'animation afin de fluidifier la navigation a été ajouté. 